Cahiers $d u$ MONDE RUSSE

\section{Cahiers du monde russe}

Russie - Empire russe - Union soviétique et États indépendants

$50 / 2-3 \mid 2009$

L'Europe orientale, 1650-1730. Crises, conflits et renouveau

\title{
Les enquêtes sur les « discours inconvenants » et l'imaginaire politique et social dans la Russie du $\mathrm{XVII}^{\mathrm{e}}$ siècle
}

"Improper language" and seventeenth-century Russian popular vision of politics and society

Pavel V. Lukin

\section{OpenEdition \\ Journals}

Édition électronique

URL : https://journals.openedition.org/monderusse/9718

DOI : 10.4000/monderusse. 9718

ISSN : $1777-5388$

Éditeur

Éditions de l'EHESS

Édition imprimée

Date de publication : 15 septembre 2009

Pagination : 337-345

ISBN : 978-2-7132-2260-3

ISSN : $1252-6576$

Référence électronique

Pavel V. Lukin, «Les enquêtes sur les « discours inconvenants » et l'imaginaire politique et social dans la Russie du xvII ${ }^{\mathrm{e}}$ siècle », Cahiers du monde russe [En ligne], 50/2-3 | 2009, mis en ligne le 13 octobre 2012, consulté le 04 septembre 2022. URL : http://journals.openedition.org/monderusse/ 9718 ; DOI : https://doi.org/10.4000/monderusse.9718 


\section{LES ENQUÊTES \\ SUR LES « DISCOURS INCONVENANTS » ET L'IMAGINAIRE POLITIQUE ET SOCIAL DANS LA RUSSIE DU XVII ${ }^{\mathrm{e}}$ SIÈCLE}

Les historiens russes ont tendance à l'heure actuelle à se concentrer sur la vie spirituelle et intellectuelle des hommes du passé - ce que l'on appelle parfois « mentalités », ou Weltanschauung, ou encore «l'imaginaire ». La façon dont le pouvoir politique, réel ou fictif, se réflète dans l'imaginaire populaire revêt une importance particulière dans ce contexte, et le XVII ${ }^{\mathrm{e}}$ siècle russe nous offre à cet égard un champ de recherche tout à fait passionnant. Suffisamment archaïque, puisqu'il s'agit de la période qui précède le règne de Pierre le Grand, elle a en même temps produit des sources abondantes qui correspondent bien au sujet que nous avons choisi.

Un certain nombre d'erreurs de méthodologie ont faussé les études consacrées jusqu'à présent à cette question. Les auteurs se représentent la mentalité des Russes du XVII ${ }^{\mathrm{e}}$ siècle comme homogène ; ce jugement a priori, s'appuyant sur un petit nombre de sources, ne prend pas garde au fait que l'imaginaire peut différer selon les groupes et les catégories sociales. De ce fait, on s'autorise l'usage de sources, par exemple les proverbes, dont l'origine sociale est très difficile, voire impossible, à déterminer. On peut regretter également la prédilection des historiens pour les sources déjà publiées concernant les mouvements sociaux.

On a vu apparaître ces derniers temps un phénomène nouveau (bien qu'on puisse lui trouver des antécédents à l'époque soviétique et même présoviétique) : l'auteur commence par échafauder une théorie globale sans véritable base documentaire, puis il s'appuie sur sa propre théorie comme si elle était déjà démontrée, donc incontestable. L'ouvrage de Andrej Jurganov, consacré aux catégories de la culture russe médiévale, fournit un bon exemple de cette histoire spéculative ${ }^{1}$, qui jouit

1. Andrej L'vovič Jurganov, Kategorii russkoj srednevekovoj kul'tury [Les catégories de la culture russe médiévale], M. : Mosk. Inst. razvitija obrazovatel’nyh sistem, Inst. « Otkrytoe ob-vo », 1998. 
d'un certain succès, comme en témoigne un récent débat dans Odissej ${ }^{2}$. Sans trop se soucier des sources, l'auteur énonce ses idées sur la synthèse russo-mongole, la genèse du despotisme à l'époque du prince Andrej de Bogoljubovo, l'absence de la notion de liberté individuelle avant Pierre le Grand, etc.

Pour éviter de tomber dans ces travers, il est essentiel pour l'historien de bien choisir ses sources et sa méthodologie. C'est ce que nous avons tenté de faire en étudiant cet ensemble de documents très intéressants que représentent les enquêtes sur les « discours inconvenants » (neprigožie reči). Le sujet a déjà été abordé au début du Xx $x^{\mathrm{e}}$ siècle : en 1911 Nikolaj Novombergskij a publié une masse de matériaux considérable, mais qui ne couvre qu'une partie des enquêtes du secrétariat de la Guerre (Razrjadnyj prikaz) pendant la première moitié du XVII ${ }^{\mathrm{e}}$ siècle (avant 1649) ${ }^{3}$. Les discours « inconvenants », ou « malséants », sont des propos que les autorités russes du $\mathrm{XVII}^{\mathrm{e}}$ siècle considéraient comme des délits de lèse-majesté [slovo i delo gosudarevo], portant atteinte à «l'honneur du souverain », et qui allaient des gaffes les plus innocentes aux menaces les plus graves (« il aurait dit: “je vais couper la gorge au tsar" ») et aux crimes contre l'État.

Le dossier d'une enquête sur une " affaire inconvenante » était constitué de tout un ensemble de documents dont le premier, et pour nous l'essentiel, était la lettre de dénonciation (izvestnaja čelobitnaja, littéralement la «supplique de dénonciation »). Cependant il arrive que cette lettre manque dans le dossier ; dans ce cas le rapport (otpiska) du gouverneur au tsar, la réponse du tsar, l'interrogatoire du dénonciateur et des témoins, les procès-verbaux des confrontations, l'extrait pour compte rendu au tsar (vypiska $v$ doklad) peuvent en tenir lieu.

Les sources de ce type sont particulièrement précieuses pour le chercheur d'abord parce que l'on y retrouve, à peu de chose près, le discours spontané des hommes du XVII ${ }^{\mathrm{e}}$ siècle, que les scribes reproduisent scrupuleusement étant donné la gravité de l'affaire. C'est là un avantage considérable par rapport aux Mémoires des étrangers qui ont visité la Russie ou aux sources imprégnées de l'idéologie officielle. Ensuite, l'auteur des « discours inconvenants » est toujours identifié avec précision : on indique son origine et sa position sociale. La fréquence des « affaires inconvenantes » et l'existence même des dossiers ne sont pas étroitement liées à tel ou tel événement historique, à une révolte par exemple : nos sources reflètent donc l'imaginaire populaire tant en temps normal que dans des situations exceptionnelles. Qui plus est, leur authenticité, leur véracité ne font aucun doute. Les buts poursuivis par les autorités sont en effet très différents de ceux de l'administration soviétique de l'époque stalinienne. $\mathrm{Au} \mathrm{XVII}{ }^{\mathrm{e}}$ siècle, le tsar voulait vraiment savoir ce qui avait été dit, et c'est dans ce sens que s'orientait l'enquête sur place.

2. « Čelovek v istorii. Russkaja kul'tura kak issledovatel'skaja problema » [L'homme dans l'histoire. La culture russe comme objet de recherches], Odissej : Russkaja kul'tura kak isledovatel'skaja problema, M. : Nauka, 2001, p. 5-64.

3. Nikolaj Jakovlevič Novombergskij, Slovo i delo gosudarevy. Processy do izdanija Uloženija Alekseja Mihajloviča 1649 goda [Paroles et actes constituant un délit de lèse-majesté. Procès antérieurs à l'Établissement d'Aleksej Mihajlovič], M., 1911, vol. I. Réimpression : M. : Jazyki slavjanskoj kul'tury, 2004, 2 vol. 
Les magistrats cherchaient à reproduire, mot pour mot, même les pires grossièretés prononcées à l'adresse du tsar. Enfin, ces pièces officielles font partie des documents publics, elles ne sont pas uniques dans leur genre, contrairement aux sources généralement utilisées pour l'étude de l'imaginaire collectif. On en a conservé un nombre assez important dans les archives pour que l'on puisse parler de source sérielle.

Aussi a-t-on peine à comprendre pourquoi les « discours inconvenants » ont été jusqu'à présent à peu près ignorés par les historiens qui ne s'y réfèrent que de façon épisodique et en citant des cas isolés, alors que ces documents constituent une catégorie à eux seuls et ne doivent donc être étudiés que de façon globale. Cet éclectisme a engendré quantité d'erreurs et continue à le faire. Par exemple, on affirme souvent que le secrétariat de la Guerre était seul habilité à traiter des affaires de lèse-majesté (c'est ce qu'ont écrit Nikolaj Novombergskij et Georgij Gustavovič Telberg, qui ont été les premiers à les étudier) ou que les sanctions pour discours inconvenants étaient prévues par l'Établissement de 1649 (Sobornoe Uloženie). On continue à tenir une comptabilité futile des imposteurs au XVII ${ }^{\mathrm{e}}$ siècle, alors que l'analyse approfondie des enquêtes démontre de façon convaincante les dimensions massives du phénomène. L'imposture était sans aucun doute un des éléments essentiels de la culture politique populaire au XVII ${ }^{\mathrm{e}}$ siècle.

La plupart des enquêtes sur les « discours inconvenants » sont conservées dans les Archives d'État des actes anciens de Russie (RGADA), soit dans les fonds des secrétariats (sing. prikaz) de Moscou, soit dans ceux des administrations locales (sing. prikaznaja izba). L’idée reçue sur le rôle privilégié du secrétariat de la Guerre dans les enquêtes sur les « discours inconvenants » n'est pas justifiée. En réalité tous les secrétariats s'en occupaient à égalité. Il est vrai que celui de la Guerre avait de larges compétences, et que de plus ses archives se sont heureusement bien conservées, c'est pourquoi la plupart des affaires qui subsistent en proviennent. À l'époque de Pierre le Grand, la rédaction des documents officiels et les pratiques judiciaires ayant été mises en ordre et régularisées, les affaires politiques ont été confiées exclusivement au secrétariat de Preobraženskoe (Preobraženskij prikaz). Mais au XVII ${ }^{\mathrm{e}}$ siècle la situation n'était pas encore réglée, aussi les enquêtes sur les « discours inconvenants » peuvent-elles se trouver dans le fond de n'importe quel secrétariat, pourvu qu'il contienne des documents de cette époque. Le fond du secrétariat de la Guerre a fait l'objet, dès le XIX ${ }^{\mathrm{e}}$ siècle, d'un excellent inventaire ${ }^{4}$, mais il n'en va pas de même pour les autres fonds. On sait qu'au XVII siècle on assemblait les documents isolés, portant souvent sur des sujets tout à fait différents, dans des rouleaux (sing. stolbec) parfois composés de dizaines d'affaires hétéroclites.

Les enquêtes sur les « discours inconvenants » nous ont servi de source principale pour décrire certains aspects de l'imaginaire populaire concernant le pouvoir

4. Opisanie dokumentov i bumag hranjaščihsja v Moskovskom arhive Ministerstva Justicii [Inventaire des documents et papiers conservés dans les archives du ministère de la Justice I, abrév. usuelle : Opisanie MAMJu, SPb., 1869 (vol. I-II), M., 1876-1921 (vol. III-XXI) ; histoire des archives du secrétariat de la Guerre, vol. V; tableau d'ensemble, vol. IX ; inventaire des fonds, vol. X-XX. 
d'État au XVII ${ }^{\mathrm{e}}$ siècle $^{5}$ : la sacralisation du pouvoir impérial, les limites de ce pouvoir dans l'imagination populaire, le phénomène des faux tsars - pour ne citer que les principaux. Mais le sujet est loin d'être épuisé pour autant. Les enquêtes sur les « discours inconvenants » peuvent contribuer à éclaircir au moins trois aspects importants de l'imaginaire social et politique du XVII ${ }^{\mathrm{e}}$ siècle.

Le premier concerne la structure sociale et la constitution des ordres [sing. soslovie], telles qu'elles se reflètent dans l'imaginaire populaire de l'époque. Évidemment, les «discours inconvenants » concernent surtout le tsar, son « honneur et majesté ». Mais les affaires de lèse-majesté peuvent aussi nous renseigner sur les nouveaux groupes sociaux alors en voie de constitution et l'attitude du peuple à leur égard. Cela suppose que l'on puisse identifier avec précision le groupe social auquel appartient l'auteur de ces propos, car il ne s'agit pas ici d'un « peuple » abstrait et amorphe : nous cherchons à saisir l'image qu'un groupe social déterminé se fait d'un autre groupe. En attendant une étude détaillée, qui nécessitera un travail considérable, on peut d'ores et déjà relever quelques sujets brûlants pour la société russe de l'époque, par exemple le groupe social à part que constituent les cosaques : leur comportement ambigu pendant le Temps des Troubles était largement discuté et donnait naissance à des images contradictoires.

$\mathrm{Au}$ mois de juin 1625, un cosaque propriétaire foncier ${ }^{6}$ (belopomestnyj) de Kozel'sk, Dioniska [Petit Denys] Fedorov, porte plainte contre le prêtre du village de Ryčonok, dans le district de Peremyšl’’, Ivan Grigor'ev :

On buvait avec Ivan Grigor'ev, le prêtre de la paroisse du prophète Élie, en payant pour l'eau-de-vie, et lui s'est mis à m'insulter, en me traitant de bandit, et moi, je lui ai dit : «Je ne suis pas un bandit, j'ai prêté serment sur la croix à notre tsar, souverain et grand prince de toute la Russie Mihail Fedorovič ». Et le prêtre m'a répondu : "Vous n'avez pas prêté serment sur la croix à notre souverain, vous autres fils de putes, mais à un porc, [s'il ne tenait qu'à vous] bientôt un tsar pourrait de nouveau faire son apparition en Ukraine à cause de vos scélératesses, mais maintenant c'est fini, fils de pute, vous ne pourrez plus trahir, comme naguère, et vous fabriquer des tsars sur mesure. $»^{7}$

La Seč', quartier général des Cosaques zaporogues, foyer de rebellion et d'agitation chronique, diffusait en permanence l'esprit de révolte. En 1650 aurait eu lieu, à

5. Pavel Vladimirovič Lukin, Narodnye predstavlenija o gosudarstvennoj vlasti v Rossii XVII V. [La perception populaire du pouvoir d'État dans la Russie du XVII ${ }^{\mathrm{e}}$ siècle], M. : Nauka, 2000.

6. Il s'agit de cosaques auxquels le gouvernement impérial avait accordé des terres, à égalité avec les militaires nobles, dans les années qui suivirent la fin du Temps des Troubles (16041613).

7. Novomberskij, Slovo i delo gosudarevy, p. 18 : « Пили де мы в Ильинскаго попа Ивана Григорьева, покупая вино, и тот де поп Иван учал меня лаять, называя вором, и я де ему сказал: “Я не вор, г[осударю] ц[арю] и в[еликому] к[нязю] М[ихаилу] Ф[едоровичу] в[сея] Р[усии] крест целовал.” И поп де молыл мне: "Не г[осударю] целовали крест, целовали вы де, бледины дети, крест свинье, ужо де у вас, бледины дети, опять на Украйне царь проявится с вашим воровством, ныне де вам, блединым детям, не по-старому воровать, царей заводить.” » 
Carev Alekseev, entre le frère d'un mousquetaire de Moscou, Minka Koževnikov, qui venait d'arriver de Koroč, et un « Circassien » [cosaque d'Ukraine] anonyme, le dialogue suivant :

Ce Minka aurait dit au cosaque : «Vos Circassiens sont rassemblés, au complet, où veulent-ils aller? » Et le Circassien de lui dire, à Minka : « À la capitale, pour vous sabrer. » Et Minka aurait alors répliqué à ce Circassien : « Chez nous à Koroč on vous attend, dès que vous arriverez à Koroč, nous serons prêts [à aller] avec vous. $»^{8}$

Au lendemain du Temps des Troubles, les cosaques avaient eux-mêmes leur opinion sur le pouvoir, qui s'efforçait de réprimer ce groupe social actif en état de rebellion larvée. Une enquête de 1624 est révélatrice à cet égard. Un esclave (holop) de Livny, Griška [Petit Grégoire] Frolov rapporte qu'un cosaque « fieffé » - pomestnyj, c'est-à-dire jouissant d'un domaine en précaire ${ }^{9}-$, Ovdejko [Petit Obadiah] Jakovlev, ayant bu de la bière de ménage (braga), aurait dit :

«Vous, les rustauds (mužiki), vous suivrez [toujours] les bojare et celui que les bojare vont suivre, mais eux [les cosaques - PL], ils vont brûler leurs bénéfices et leurs patrimoines, et eux [les paysans], où iront-ils se fourrer? ». Et Ovdejko a dit qu'ils sont environ cinq cents dans le complot, et qu'ils vont se procurer un autre souverain. ${ }^{10}$

Rien d'étonnant si l'image des cosaques dans les autres couches de la société était parfois négative. En 1640 un citadin (posadskij čelovek) de Meščevsk, Fedor Blestin, aurait déclaré lors d'une beuverie, en se disputant avec des cosaques : «Le Grand Prince est bien bête de vous donner à boire et à manger, à vous autres cosaques. ${ }^{11}$

8. RGADA (Rossijskij gosudarstvennyj arhiv drevnih aktov - Archives d'État des actes anciens de Russie), f. 210 [archives du secrétariat de la Guerre], Prikaznyj stol [Bureau de service, chargé notamment de la justice et de la police dans les villes relevant du secrétariat], $\mathrm{n}^{\circ} 2172,1.57-58$ : « И говорил де тот Минка черкашенину : “Черкасы де у вас в зборе, и куды они хотят итти ?” И черкашенин говорил ему, Минке : “Будем де мы к вам до столицы вас рубить.” И он де, Минка, против тех речей говорил тому черкашенину : “А у нас де на Корочи вас ждут, а как де придете под Корочю, и мы де с вами ж готовы.”»

9. Le domaine en précaire, dont le statut rappelle le beneficium carolingien, était octroyé aux nobles, serviteurs militaires du tsar, et à quelques autres catégories de la population, sous condition de service. Aussi pouvait-il être confisqué en cas de désertion ou d'absence de descendant mâle, contrairement aux patrimoines, qui étaient la propriété héréditaire des lignages.

10. RGADA, f. 210, Dopolnitel’nyj otdel [Section complémentaire], n 23, 1. 21-22 ; «"Вы де, мужики, поидете за московских бояр и за ково они поидут, и они те их поместья и вотчины выжгут, а им самим где детца.” И Овдейко молыл, есть их в заговоре человек с пятьсот, и они себе наидут иного государя. »

11. Novombergskij, Slovo i delo gosudarevy, p. 498 : « Глуп де князь великий, что вас, казаков, поит и кормит ». 
Nos sources témoignent aussi des contradictions dans le milieu des gentilshommes, qui était loin d'être homogène. C'est ainsi qu'en 1682, un petit noble de province (syn bojarskij) de la ville d'Elec, venu à Moscou au secrétariat de la Guerre pour y déposer une supplique, aurait dit :

Les bojare et les gentilshommes de Moscou sous Čigirin n'ont pas fait leur devoir militaire ; pendant la bataille ils se sont abrités sous des charrettes et dans des trous, et on n'en a pas tué beaucoup, de ces putains, désormais eux [les gentilshommes de province - PL] vont les battre à coups de gourdin jusqu'à la mort ; les braves ont été tués, et eux, ces vilaines putes, ont échappé à la mort. ${ }^{12}$

Les enquêtes sur les « discours inconvenants » reflètent, de même, les antagonismes, typiques de la première moitié du XVII ${ }^{\mathrm{e}}$ siècle, entre les habitants des enclaves privilégiées (sing. sloboda, litt. « franchise », « sauveté »), dites «blanches », et les citadins taillables des quartiers « noirs » d'une part, et d'autre part entre l'ensemble des habitants des villes, unis cette fois contre les militaires roturiers, surtout les mousquetaires (sing. strelec) - antagonismes auxquels les historiens n'ont pas prêté suffisamment attention jusqu'à présent, et qui soulèvent une question importante : comment les gens du XVII siècle conciliaient-ils la hiérarchie sociale avec l'idée chrétienne de l'égalité de tous les mortels, frères « par la chair », devant la face de Dieu ?

Le second aspect concerne le rôle de l'Église et du clergé dans la vie de l'État et de la société. Comment apparaît l'Église, dont l'influence au XVII siècle ne saurait être surestimée, dans l'imaginaire populaire ? Comment les laïcs des différentes couches sociales comprennent-ils le rôle du clergé dans la société ? Et, symétriquement, quelles sont les idées du clergé en matière sociale et politique ? Plus précisément, que pensent les ecclésiastiques du clergé et des relations entre le pouvoir ecclésiastique et le pouvoir séculier?

Parfois on trouve des témoignages d'une attitude nettemment méprisante à l'égard des prêtres. Par exemple, en 1625, dans le hameau de Sel'co près de Brjansk, Nehorošij, fils de Neždan, Semičev, petit noble de province, aurait déclaré en présence du prêtre Ivan (qui avait commencé par se rendre dans un autre village que le sien) : «Quand le pope craint quelqu'un, c'est chez lui qu'il va en premier ; nous, il ne nous craint pas, il faut donc lui fracasser sa calvitie ». Intimidé, le prêtre avale l'injure, bien que son frère, diacre dans la même église, ait essayé de protester, disant que, sans la volonté de Dieu et du « grand souverain, le patriarche Philarète ${ }^{13} »$, on n'avait pas le droit de battre un prêtre. Sur quoi Semičev rétorque : «Moi, je crache sur Philarète ! ${ }^{14}$.

12. RGADA, f. 210, rouleaux du bureau de Belgorod, $\mathrm{n}^{\circ}$ 1045, f. 27 : « Бояря де и московских чинов люди, будучи под Чигирином, не служили, толко де з боем хоронились под телеги и в ямы, а ныне де из них, таких матерей, побито немного, а впредь де они их станут бить ослопьем до смерти, добрые де побиты, а лихие такие матери остались. »

13. Père du tsar Mihail Fëdorovič et chef de l'Église orthodoxe de Russie.

14. Novombergskij, Slovo i delo gosudarevy, p. 16 : «Поп де кого блюдется, к тому поперед ходит, а нас де не блюдется надобно де попу плешь разбить». 
De son côté, au cours de la seconde moitié du XVII ${ }^{\mathrm{e}}$ siècle, le clergé prend conscience de lui-même comme groupe social, conscience qui transparaît, par exemple, dans les propos « inconvenants » de Lavrentij, trésorier du couvent (féminin) de l'Intercession de la Vierge de Suzdal'. Le trésorier jugeait un sous-secrétaire (pod'jačij) du même monastère, Nazar Ivanov, pour viol d'une religieuse. Pendant le procès l'accusé se fait accusateur : Lavrentij se serait rendu coupable de lèse-majesté. «Lui, Nazar, a demandé au père Lavrentij que le procès soit mené « conformément aux édits (sing. ukaz) du grand souverain », et le trésorier Lavrentij lui aurait répondu : « chez nous [le clergé] nous n'obéissons pas aux édits du souverain ni à l'Établissement des États [de 1649], mais nous avons nos édits à nous, ceux de nos prélats ». C'est là une situation vraiment originale. Le sous-secrétaire accusé de viol essaye d'échapper au châtiment en faisant référence à l'autorité suprême, celle du tsar, et à sa volonté, exprimée à travers les « édits du souverain » et le Code de 1649. Mais son juge, le trésorier Lavrentij, refuse d'obéir à la juridiction du pouvoir séculier ${ }^{15}$. En réalité, il ne fait que constater les faits : le viol relevait en effet du tribunal ecclésiastique. Mais dans ce contexte « les édits du souverain » et ceux de l'Église sont évidemment ressentis comme antagonistes.

Le troisième aspect de la recherche, le plus riche peut-être, mais aussi le plus difficile à mettre en œuvre, consiste à explorer l'opinion publique du XVII ${ }^{\mathrm{e}}$ siècle, autrement dit la perception populaire des événements politiques. Bien sûr, « opinion publique » n'est pas le mot juste. Il s'agit plutôt de « bruits », de « rumeurs », pour reprendre le titre de l'article de Sergej Bahrušin ${ }^{16}$. En abordant cet ensemble de problèmes, il faut en premier lieu distinguer deux faces distinctes du phénomène. Il y a ce que l'on pourrait appeler le «bruit de fond », c'est-à-dire l'image que se faisaient les hommes du XVII ${ }^{\mathrm{e}}$ siècle de la société et de la vie politique en l'absence de toute situation extraordinaire. C'est seulement si l'on en tient compte que l'on peut interpréter, sans risque d'erreur, l'autre versant de l'opinion publique : les réactions ponctuelles des contemporains aux événements historiques ou aux décisions des gouvernants. De ce point de vue, les enquêtes sur les « discours inconvenants » représentent une source précieuse, et presque la seule, sur ce que les Russes $\mathrm{du} \mathrm{XVII}{ }^{\mathrm{e}}$ siècle pensaient et disaient du pouvoir politique, et, plus largement, sur les mécanismes de formation de l'opinion publique, ou de ce qui en tenait lieu, dans une société traditionnelle.

Il est impossible de dresser une statistique de ce genre de discours, et cela d'autant plus que les dénonciateurs se montraient très pointilleux sur l'honneur de leur souverain. Il s'agit de jugements formulés sur tel ou tel aspect de la politique des tsars, de propos (en majorité péjoratifs) à l'adresse des favoris de toutes sortes :

15. RGADA, f. 210, rouleaux du bureau de Belgorod, $\mathrm{n}^{\circ}$ 143, 1. $413:$ « У нас де не государев указ и не соборное уложенье, свой светительской указ ».

16. Sergej Vladimirovič Bahrušin, «Političeskie tolki v carstvovanie Mihaila Fëdoroviča » [Rumeurs à caractère politique sous le règne de Mihail Fëdorovič], in Trudy poistočnikovedeniju, istoriografii $\mathrm{i}$ istorii Rossii perioda feodalizma [Travaux d'étude des sources, d'historiographie et d'histoire de la Russie pendant la période du féodalisme], M. : Nauka, 1987, p. 87-118. 
le patriarche Philarète, la nonne Marfa ${ }^{17}$, Boris Ivanovič Morozov ${ }^{18}$. Les Russes de l'époque aimaient faire des potins sur la vie privée des membres de la famille du tsar. Les gens simples discutaient aussi des problèmes de politique extérieure (surtout des relations compliquées avec la Crimée et le «tsar des Turcs », avec la Rzecz Pospolita, la « république » polonaise). Les habitants des régions limitrophes étaient à peu près au courant des événements à l'étranger, ils pouvaient comparer des sociétés aussi différentes que celles, par exemple, de Russie et de Lituanie.

C'est ainsi que le prisonnier Ivaška [Jeannot] Lukin, détenu à Galič, aurait dit « de la très-chrétienne souveraine, tsarine et grande princesse Natal'ja Kirillovna ${ }^{19}$ qu'elle faisait des avances au roi de Pologne et voulait l'épouser »; l'autre prisonnier, Kuska [Petit Kuz'ma, c'est-à-dire Côme] Osipov, « en réponse à cela aurait dit : "il est temps de pendre tous les traîtres (les Naryškin - PL)" ». ${ }^{20}$ Ce dialogue montre bien comment les masses populaires percevaient la vie privée des membres du clan au pouvoir et la lutte acharnée au sommet entre les factions de la Cour.

L'analyse des enquêtes sur les « discours inconvenants » pourrait aussi contribuer à nous familiariser avec d'autres sources du même type. C'est le cas notamment des blasphèmes et autres délits religieux classés sous la rubrique « affaires d'hérésie » : il s'agit de dossiers concernant des personnes accusées de paroles ou d'actes non conformes à la doctrine ou aux pratiques de l'Église officielle. Les « affaires d'hérésie » du XVIII" siècle ont été étudiées par Elena Smiljanskaja ${ }^{21}$, mais rien n'a été fait pour le $\mathrm{XVII}^{\mathrm{e}}$ siècle, qui reste de ce point de vue une tabula rasa. Or l'étude des « affaires d'hérésie » peut utilement compléter nos connaissances dans de nombreux domaines, explorés depuis longtemps par les chercheurs occidentaux et tout récemment par les historiens russes. En ce qui concerne ces derniers, toutefois, ce sont surtout des documents isolés, principalement littéraires, qui ont servi de source. Les « affaires d'hérésie » portent sur des aspects très variés du sentiment religieux populaire : la perception des saints et de la sainteté en tant que telle, l'imaginaire eschatologique, les « fols en Christ $^{22}$ » (jurodivye) - phénomène

17. Mère du tsar.

18. Gouverneur d'Aleksej, le prince héritier, il était devenu l'un des plus riches propriétaires terriens de Russie.

19. Natal’ja Kirillovna, née Naryškina, 1651-1694. Seconde épouse du tsar Aleksej Mihajlovič et mère de Pierre le Grand.

20. RGADA, f. 210, « Bureau des ordres », rouleau n 658, 1. 96-97 : « про благоверную государыню царицу и великую княгиню Наталию Кириловну говорил, что де бутто она подзывает короля полского и хочет за него итти замужь » ; « против того говорил: “Пора де изменников перевешать." »

21. Elena Borisovna Smiljanskaja, Volšebniki, bogohul'niki, eretiki : narodnaja religioznost' $i$ «duhovnye prestuplenija » V Rossii XVIII V. [Magiciens, blasphémateurs, hérétiques : piété populaire et « délits sacrés » en Russie au XVIII siècle], M. : Indrik, 2003.

22. Voir sur ce point les travaux controversés, mais intéressants, de Sergej Arkad'evič Ivanov, Vizantijskoe jurodstvo [Les fols en Christ à Byzance], M. : Meždunarodnye otnošenija, 1994, et Blažennye pohaby: Kul'turnaja istorija jurodstva [Obscénités bienheureuses: Histoire culturelle des fols en Christ], M. : Jazyki slavjanskih kul'tur, 2005 (trad. angl. de Simon Franklin, Holy Fools in Byzantium and Beyond, New York : Oxford University Press, 2006). 
presque aussi répandu, à notre avis, que les faux tsars, étroitement lié de surcroît à l'idéologie sociale et politique des masses populaires — , la démonologie, l'attitude envers les icônes et autres objets sacrés, les représentations de l'au-delà (et dans ce cas les « affaires d'hérésie » peuvent être combinées avec les « visions », que l'on met habituellement à contribution pour traiter ce sujet). Non contents d'être une source féconde, dont nous sommes loin d'avoir exploité toutes les possibilités, les enquêtes sur les « discours inconvenants » pourraient ainsi servir de propédeutique à de nouvelles façons d'écrire l'histoire.

Académie des sciences de Russie, Institut d'histoire russe, Moscou

lukinpavel@yandex.ru 